\title{
A Case of Trauma-Induced Arterial Thrombosis Mimicking Vasculitis
}

\author{
${\text { Tanureet } \text { Kochar }^{1} \text {, } \text { Khalil Bourji }^{2} \text {, Housam Sarakbi }}^{2}$ \\ 1. Sleep Medicine, Wayne State University Detroit Medical Center, Detroit, USA 2. Rheumatology, Wayne State \\ University Detroit Medical Center, Detroit, USA
}

Corresponding author: Tanureet Kochar, tanureet.kochar27@gmail.com

\begin{abstract}
Vasculitis mimickers are structural or pathologic entities that resemble the vasculitis clinical presentation and/or diagnostic findings. Their presence can be a conundrum, and physicians require careful assessment and adequate knowledge physicians when considering a diagnosis of vasculitis. Although they are considered mimickers, the therapeutic approach for most of them differs widely from that of vasculitis as high-dose steroids and potent immunosuppressive regimens are usually indicated in the latter. In fact, steroid therapy is contraindicated and is considered harmful in some of these mimickers (e.g. segmental arterial mediolysis). Therefore, it is important to distinguish them from vasculitis to prevent complications from immunosuppressive therapy. Hereby, we present a challenging case of a 64-year-old man who presented with acute gangrenous changes on his right fingers due to arterial thrombus after trauma resembling vasculitis.
\end{abstract}

Categories: Rheumatology

Keywords: vasculitis, trauma, vasculitis mimickers, thrombosis, arterial

\section{Introduction}

Vasculitis has a wide range of presentations, from only cutaneous manifestation to multisystem organ involvement. There are many medical conditions that mimic vasculitis and have been known to cause a vasculitis-like picture. These include infections, drugs, and malignancy [1]. Miloslavsky et al. stated the following nine medical conditions as challenging primary vasculitis mimickers: fibromuscular dysplasia, calciphylaxis, segmental arterial mediolysis, antiphospholipid syndrome, hypereosinophilic syndrome, lymphomatoid granulomatosis, malignant atrophic papulosis, livedoid vasculopathy, and immunoglobulin G4-related disease [2]. Their presence can be a conundrum, and physicians require careful assessment and adequate knowledge when considering a diagnosis of vasculitis. Although they are considered mimickers, the therapeutic approach for most of them differs widely from that of vasculitis, as high-dose steroid and potent immunosuppressive regimens are usually indicated in the latter. In fact, steroid therapy is contraindicated and is considered harmful in some of these mimickers (e.g. segmental arterial mediolysis)

Received 08/10/2020

Review began 09/01/2020 Review ended 09/17/2020 Published 09/21/2020

\section{() Copyright 2020}

Kochar et al. This is an open access article distributed under the terms of the Creative Commons Attribution License CC-BY 4.0., which permits unrestricted use, distribution, and reproduction in any medium, provided the original author and source are credited.
[3]. Arterial thrombus developing after trauma can present as gangrene in the distal territory of the affected artery. The acuity and the clinical manifestation of arterial thrombosis can mimic vasculitis. It is prudent to distinguish the vasculitis mimickers from true vasculitis to prevent unnecessary immunosuppressive therapy, which has its own complications $[1-2,4]$.

\section{Case Presentation}

A 64-year-old Caucasian man with a past medical history of hypertension with an unremarkable history of cardiovascular diseases or other major risk factors (tobacco use, diabetes, dyslipidemia, or family history), presented to the emergency department (ED) with pain and cold sensation in the second, third, and fourth fingers of his right hand. He reported that he was chopping a tree three days prior and accidentally hit his right arm against a big rock at the base of that tree. He initially felt pain in his fingers, which was relieved by putting ice-cold packs, but two days later, he noticed a change in the color of his fingers to purple so he decided to come to the ED. In the ED, his vital signs showed elevated blood pressure (160/94 mmHg), normal heart rate $(70 \mathrm{bpm})$, normal pulse oxygenation level ( $97 \%$ on room air), and normal body temperature. His physical examination revealed well-demarcated purple tips of the right hand's second and thirds digits distal to the distal interphalangeal (DIP) joints (Figure 1) and decreased pulses of the right radial artery and right dorsalis pedis; otherwise, his exam was unremarkable. His lab workup showed a normal complete blood count (CBC) except for mild leukocytosis $(12.6 \mathrm{~K} / \mathrm{cumm})$ and elevated creatinine $(1.48 \mathrm{mg} / \mathrm{dl})$. Thrombophilia and coagulation studies showed normal active partial thromboplastin time (aPTT), slightly elevated prothrombin time (PT) at $12.3 \mathrm{sec}$ (normal range 9.4-11.2 sec), borderline high international normalized ratio (INR) at 1.2 (normal range $0.9-1.13$ ), normal protein S activity, borderline low protein C activity at 64\% (normal range 73-144\%), normal antithrombin III antigen, absence of Factor V Leiden mutation, negative anticardiolipin antibodies, lupus anticoagulant, and beta-2-glycoprotein antibodies. His serology showed weakly positive antinuclear antibodies (ANA) with a titer of 1:80 but negative for antidouble strand DNA (dsDNA), rheumatoid factor (RF), anti-neutrophil cytoplasmic antibodies (ANCA), complement $\mathrm{C} 3$ and $\mathrm{C} 4$, erythrocyte sedimentation rate (ESR), and C-reactive protein (CRP). 


\section{Cureus}

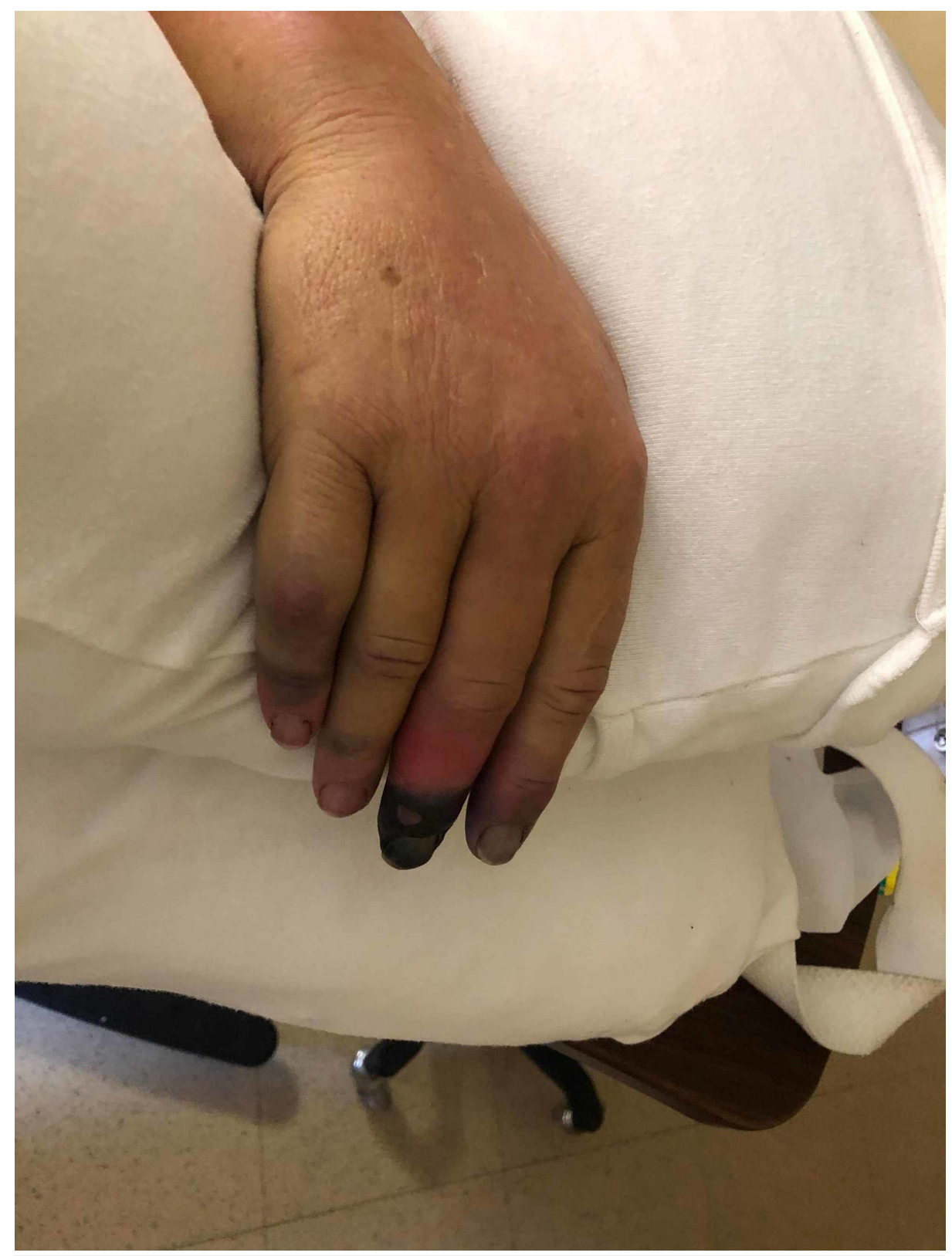

\section{FIGURE 1: Gangrene at the right middle finger}

Other diagnostic studies included normal electrocardiogram and transthoracic echocardiogram, without evidence of arrhythmias or cardiac thrombi. Computed tomography angiography (CTA) of the right upper extremity revealed short segment intraluminal thrombus $2 \mathrm{~cm}$ below the elbow joint within the right brachial artery, with diffuse vasospasm distally (Figure 2). Arterial Doppler study showed no flow to the distal second and third digits of the right hand (Figures 3-4). Due to a decrease in the right dorsalis pedis pulse, CTA of the abdomen, pelvis, and lower extremities was done and showed normal aorta and major branches, with mild atherosclerotic calcification in the femoral artery and occlusion of the anterior tibial artery at its origin. 


\section{Cureus}

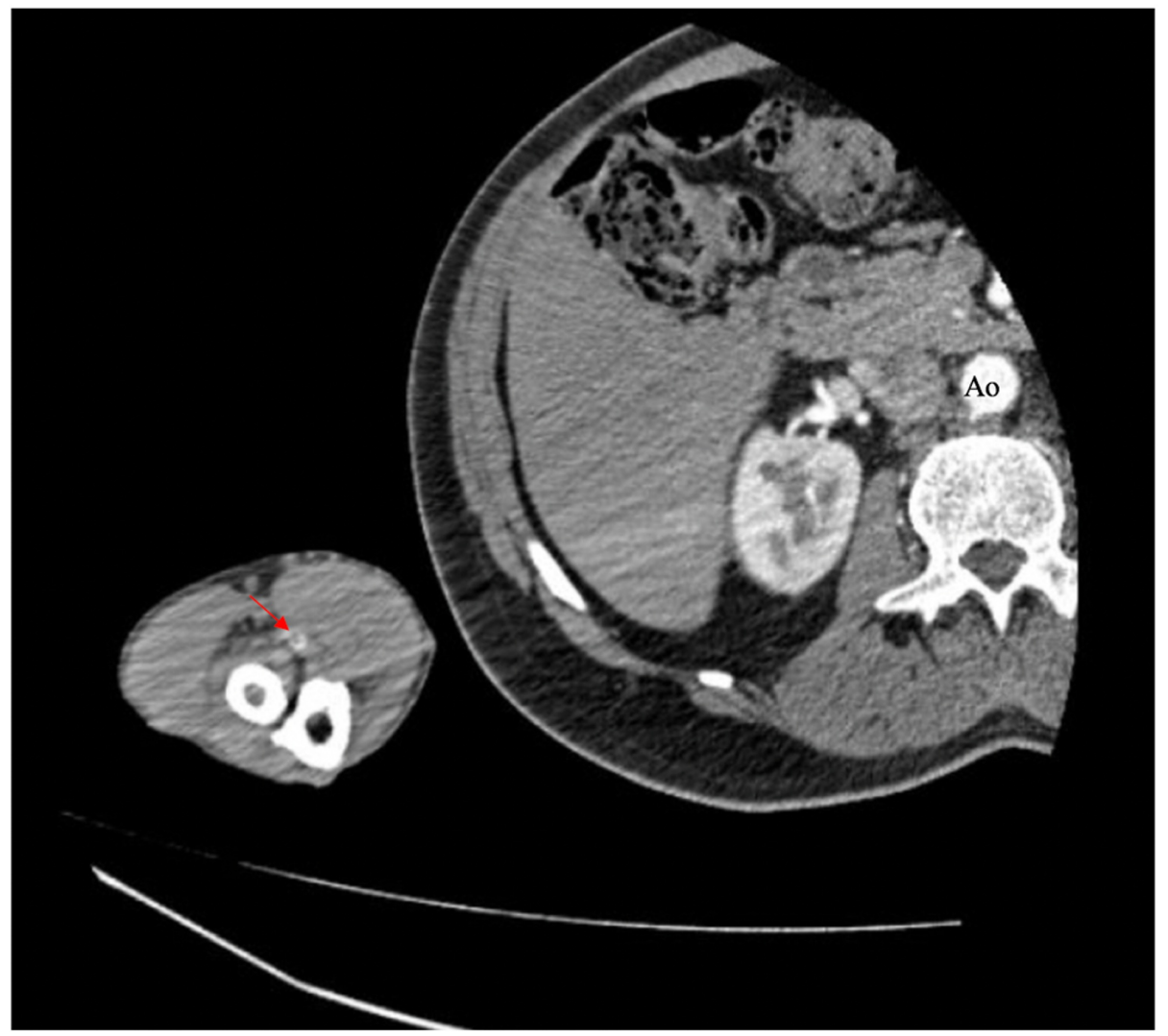

FIGURE 2: Computed tomography angiography (CTA) of the right-hand revealing thrombus formation (red arrow) and filling defect as compared to the aorta (Ao) 


\section{Cureus}
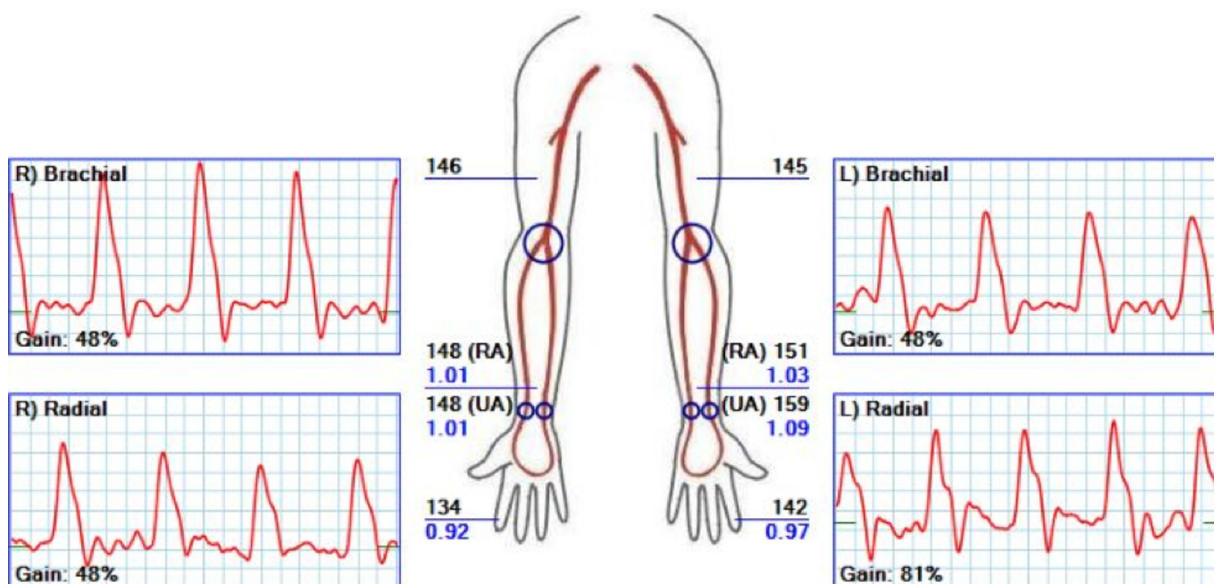

1.01

Gain: $48 \%$

Gain: $48 \%$

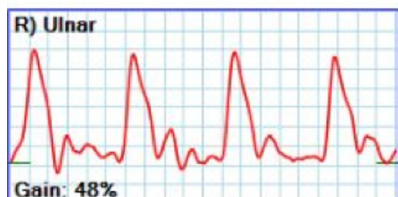

1.01 Wrist/Brachial Index

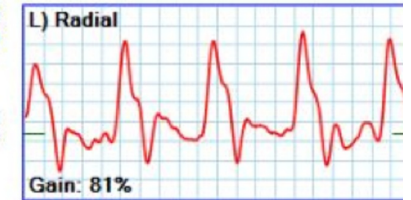

R) Digit
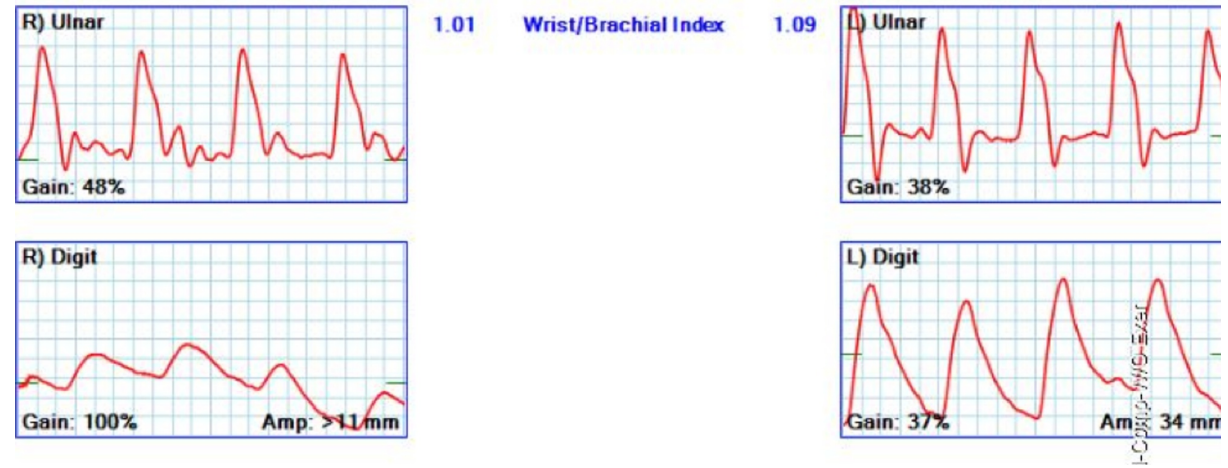

FIGURE 3: Segmental blood pressure and digital/brachial indexes 


\section{Cureus}
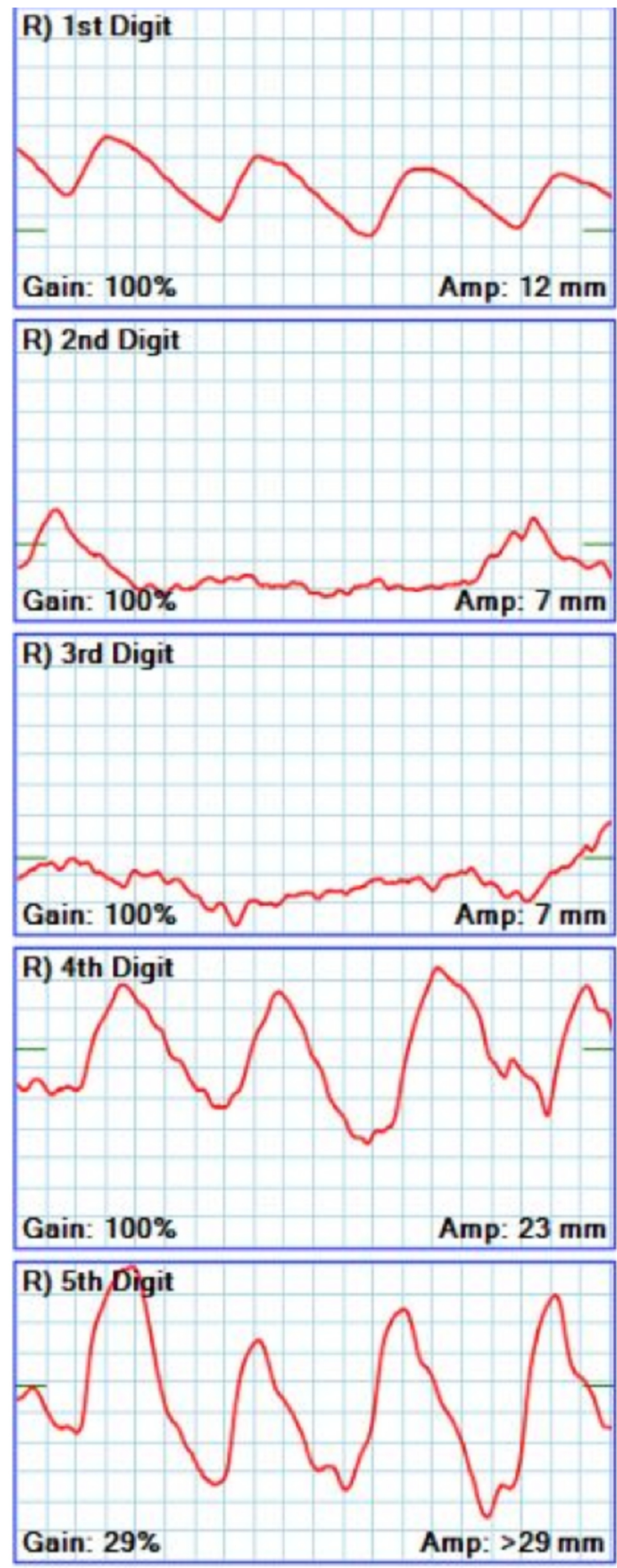

FIGURE 4: Right-hand Doppler blood flow waveform with normal triphasic waves in the first, fourth, and fifth digits and flat line in the second and third digits

\section{Management}

The patient was admitted to the hospital and started on aspirin and statin and was given an intravenous heparin drip for four days. After the aforementioned extensive workup that showed negative findings for systemic vasculitis or prothrombotic state, the patient was treated with nifedipine, followed by right stellate sympathetic ganglion block on days five and nine. Throughout the hospital stay, the patient continued to have a palpable radial pulse and Dopplerable ulnar signals and palmar arch signals. He was discharged home on the 13th day with aspirin, statin, and Xarelto. 


\section{Discussion}

Our case highlights the rare possibility of developing arterial thrombus after trauma, which mimics vasculitis. Although venous thromboembolism and deep venous thrombosis are known complications after trauma, arterial thrombus after trauma is rarely reported in the literature [5]. Initially, in this case, the cause of thrombosis was thought to be vasculitis but with further laboratory testing and imaging, vasculitis was ruled out. Although the patient had weakly positive ANA (titer 1:80) the rest of the workup, including serologic and inflammatory markers and complement levels, was normal, making vasculitis less likely. Other potential causes of thrombus, such as cardioembolism, were ruled out since there was no evidence of arrhythmia or intracardiac thrombus on electrocardiogram and echocardiogram, respectively. Arterial imaging revealed no evidence of atherosclerosis, and hypercoagulability was ruled out as well. Although any form of vasculitis, whether large, medium, or small-vessel vasculitis, can present with digital ischemia and gangrene, the lone manifestation of ischemia and gangrene is uncommon [6].

The diagnosis of vasculitis, in general, is challenging since the differential diagnosis is broad and it can have various manifestations. Vasculitis mimickers must be excluded first. Infections such as hepatitis B and C, human immunodeficiency virus (HIV), and tuberculosis (TB) are important secondary causes of vasculitis; therefore, they must be excluded [7-9]. Hematological malignancies, such as myeloproliferative and lymphoproliferative disorders, solid tumors, and drugs, should be ruled out. A thorough history, physical examination, and a battery of laboratory testing and imaging can all help in differentiating one from another [10].

Post-traumatic venous thromboembolism and deep venous thrombosis have been well-described in the literature but arterial thrombosis after trauma is a rare entity [11]. The mechanism of endothelial injury for arterial thrombosis differs from that of venous thrombosis. Underlying atherosclerosis or hypertension, which creates turbulence in the blood flow, ultimately resulting in the activation of platelets and the formation of a plaque, is thought to play a key role in arterial thrombus formation [12].

To the best of our knowledge, this is the first case in the literature that presented with a vasculitis-like picture after trauma. Karaarslan et al. have reported a case of ulnar arterial thrombosis after a trauma that presented with hand pain and swelling rather than digital gangrene [13]. "Hypothenar-hammer" syndrome has been commonly described in the literature, which refers to an injury to the ulnar artery as a consequence of repetitive trauma, which results in thrombosis and embolism of digital arteries. It is most commonly seen in athletes (hockey players, karate experts) and some occupations such as machinery, carpentry, or any industry requiring repeated striking of tools. However, arterial thrombosis after single blunt trauma is rarely described [14-15].

\section{Conclusions}

Post-traumatic arterial thrombosis are uncommon, and their clinical presentation can mimic systemic vasculitis. Vasculitis mimickers have been reported and usually create a real conundrum to clinicians. In the context of arterial thrombosis with gangrene, it is crucial to consider and rule out other etiologies, including systemic vasculitis, as the therapeutic approach varies widely and a misdiagnosis can lead to life-threatening outcomes. Therefore, vasculitis mimickers should also be ruled out as a potential cause of gangrene.

\section{Additional Information \\ Disclosures}

Human subjects: Consent was obtained by all participants in this study. Conflicts of interest: In compliance with the ICMJE uniform disclosure form, all authors declare the following: Payment/services info: All authors have declared that no financial support was received from any organization for the submitted work. Financial relationships: All authors have declared that they have no financial relationships at present or within the previous three years with any organizations that might have an interest in the submitted work. Other relationships: All authors have declared that there are no other relationships or activities that could appear to have influenced the submitted work.

\section{References}

1. Suresh E: Diagnostic approach to patients with suspected vasculitis. Postgrad Med J. 2006, 82:483-488. 10.1136/pgmj.2005.042648

2. Miloslavsky EM, Stone JH, Unizony SH: Challenging mimickers of primary systemic vasculitis . Rheum Dis Clin North Am. 2015, 41:141-160.

3. Tie JT: Systemic, cerebral, and pulmonary segmental mediolytc arteriopathy: Villainous masqueraders of vasculitis. Cardiovasc Pathol. 1996, 5:305-314. 10.1016/S1054-8807(96)00071-3

4. Zarka F, Veillette C, Makhzoum JP: A review of primary vasculitis mimickers based on the Chapel Hill Consensus Classification. Int J Rheumatol. 2020, 2020:8392542. 10.1155/2020/8392542

5. Ruskin KJ: Deep vein thrombosis and venous thromboembolism in trauma . Curr Opin Anaesthesiol. 2:215218. 10.1097/ACO.0000000000000567

6. Lau RA, Bains R, Suraweera D, Ma J, Heinze ER, Wong AL, Clements PJ: A rare case of digital ischemia and gangrene in ANCA-associated vasculitis with review of the literature. 2017, 2017:2421760. 


\section{Cureus}

$10.1155 / 2017 / 2421760$

7. Sansonno D, Dammacco F: Hepatitis C virus, cryoglobulinaemia, and vasculitis: immune complex relations . Lancet Infect Dis. 2005, 5:227-236. 10.1016/S1473-3099(05)70053-0

8. Chetty R: Vasculitides associated with HIV infection. J Clin Pathol. 2001, 54:275-278. 10.1136/jcp.54.4.275

9. Guillevin L, Mahr A, Callard P, et al.: Hepatitis B virus-associated polyarteritis nodosa. Clinical characteristics, outcome, and impact of treatment in 115 patients. Medicine (Baltimore). 2005, 84:313-322. 10.1097/01.md.0000180792.80212.5e

10. Gross W L, Trabandt A, Reinhold-Keller E: Diagnosis and evaluation of vasculitis . Rheumatology. 2000, 39:245-252. 10.1093/rheumatology/39.3.245

11. Knudson MM, Ikossi DG, Khaw L, Morabito D, Speetzen LS: Thromboembolism after trauma: an analysis of 1602 episodes from the American College of Surgeons National Trauma Data Bank. Ann Surg. 2004, 240:490-498. 10.1097/01.sla.0000137138.40116.6c

12. Rumbaut RE, Thiagarajan P: Arterial, venous, and microvascular hemostasis/thrombosis . Platelet-Vessel Wall Interactions in Hemostasis and Thrombosis. 2010 (ed): Morgan \& Claypool Life Sciences, San Rafael (CA);

13. Karaarslan AA, Karakaşlı A, Mayda A, Karcı T, Aycan H, Kobak S: Traumatic distal ulnar artery thrombosis. Case Rep Orthop. 2014, 2014:983160. 10.1155/2014/983160

14. De Monaco D, Fritsche E, Rigoni G, Schlunke S, Von Wartburg U: Hypothenar hammer syndrome: retrospective study of nine cases. J Hand Surg. 1999, 24B:731-734. 10.1054/JHSB.1999.0248

15. Vayssairat M, Debure C, Cormier J, P. Bruneval, C. Laurian, Juillet Y: Hypothenar hammer syndrome: seventeen cases with long-term follow-up. J Vasc Surg. 1987, 5:838-843. 10.1016/0741-5214(87)90096-6 